\title{
Sichere Diagnose und schnelle Reperfusion Akut-Management beim Myokardinfarkt
}

\author{
M. P. Heintzen, Medizinische Klinik II, Klinik für Kardiologie, Pneumologie, Angiologie, Klinikum Braunschweig
}

NOTFALLMEDIZIN 2003; 29: 448-452

Entscheidend für das Akut-Management von Patienten mit Myokardinfarkt ist die schnelle und sichere Diagnostik, die korrekte Risikostratifizierung und die schnellstmögliche Einleitung der adäquaten Therapie.

Die Basistherapie des Myokardinfarktes (NSTEMI und STEMI) beinhaltet Analgesie, Sedierung, Thrombozytenfunktionshemmung, Antikoagulation, Sauerstoffgabe und Betablockertherapie. Bei STEMI ist die Infarktgefäßrevaskularisation primäres Ziel. Insbesondere in den ersten drei Infarktstunden, eventuell bereits prästationär durch Thrombolyse, wenn verfügbar besser durch Kathetertherapie. Die Katheterbehandlung ist bei STEMI die erfolgreichste Revaskularisationsmaßnahme, steht jedoch nur in begrenztem Umfang zur Verfügung. Durch den Aufbau regionaler Kooperationsnetzwerke kann eine interventionelle Behandlung von sehr vielen Patienten mit STEMI erreicht werden, sodass die flächendeckende Etablierung derartigen Versorgungsstrukturen zwischen Krankenhäusern ohne Katheterlabor und erfahrenen interventionellen Zentren sinnvoll und notwendig ist. Auch Patienten mit NSTEMI profitieren von einer frühzeitigen (binnen 24 Stunden) invasiven Diagnostik und interventionellen Therapie, sodass auch diese Patienten im Rahmen eines derartigen Netzwerks betreut werden sollten.

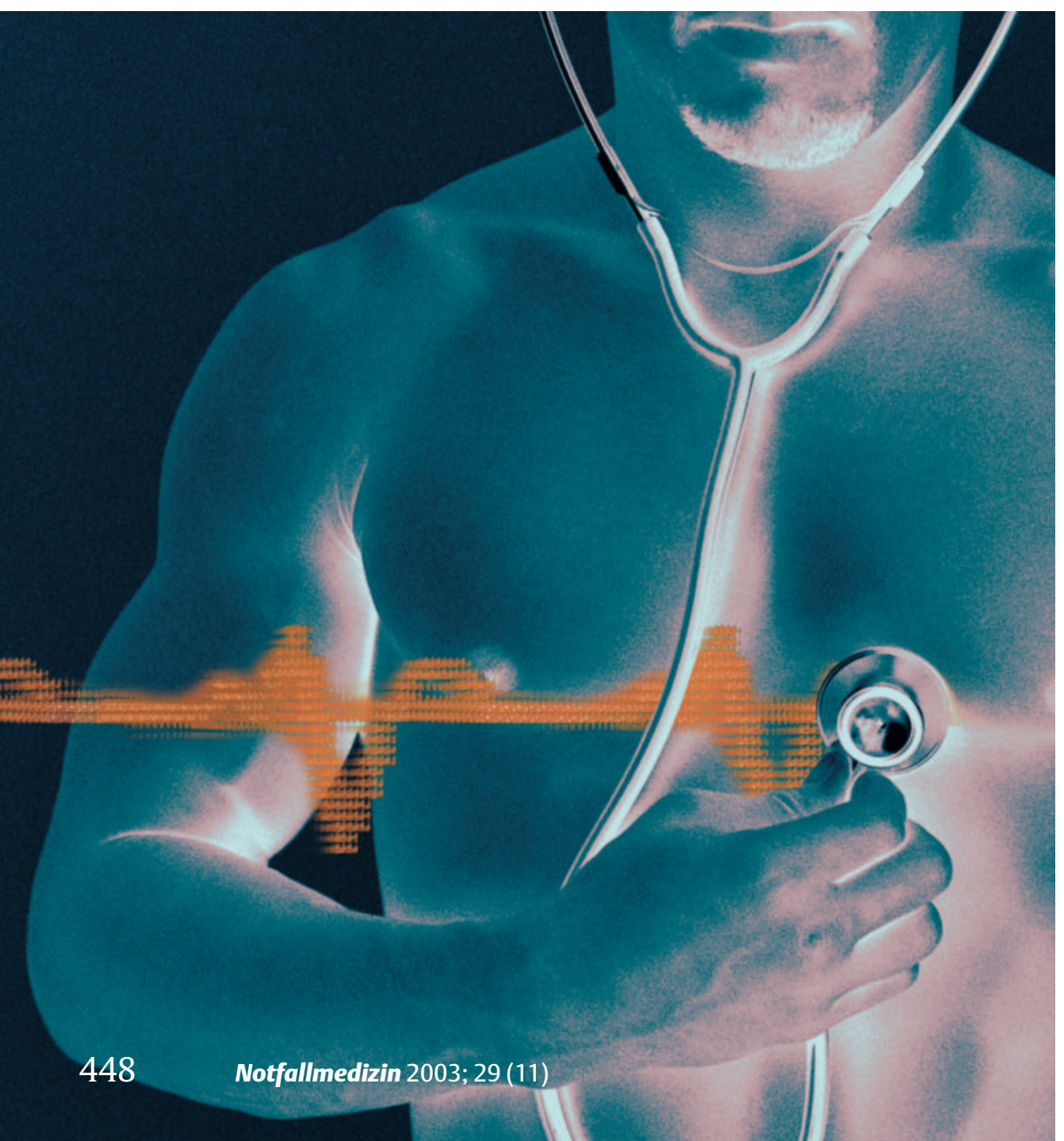

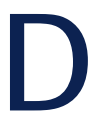
ie Überlebenswahrscheinlichkeit von Patienten mit akutem Myokardinfarkt ist in starkem Maße abhängig von der Größe des Infarktes und der Zeit, die seit Infarktbeginn verstreicht. Vor allem die gezielte frühestmögliche Therapie bewirkt eine deutliche Verbesserung der Prognose von Patienten mit Myokardinfarkt. Für das Akut-Management von Patienten mit akutem Myokardinfarkt sind daher die sichere und schnelle Diagnose und daran anschließend die sofortige komplette Reperfusion des Infarktareals von zentraler Bedeutung. Die Bemühungen um die pharmakologische oder mechanische Reperfusion des Infarktgefäßes werden in der Frühphase des Infarktes von einer auf die pathophysiologischen Grundlagen des Myokardinfarktes gerichteten modernen adjuvanten Pharmakotherapie begleitet. Gleichzeitig müssen im Rahmen des intensivmedizinischen Monitorings die möglichen Infarktkomplikationen erfasst und behandelt werden.

\section{Diagnostik}

Patienten mit akutem Thoraxschmerz werden auf einer Notaufnahmestation (in Zukunft hoffentlich vermehrt in spezialisierten „Akuten-Thoraxschmerz-Stationen“, „Chest-Pain-Units“) oder der Intensivstation vordringlich, zielgerichtet und schnellstmöglich mit dem Ziel des Nachweises oder Ausschlusses eines koronar bedingten Thoraxschmerzes untersucht. Dabei werden neben der Vielzahl von denkbaren Differentialdiagnosen vor allem die prognostisch besonders relevanten Erkrankungen (Lungenarterienembolie, Aortendissektion und Pneumothorax) in die ersten Überlegungen mit einbezogen.

Unmittelbar nach stationärer Aufnahme wird parallel zur Anam- 
neseerhebung und körperlichen Untersuchung ein 12-Kanal-EKG abgeleitet, ein venöser Zugang gelegt und Blutproben zur Analyse einer myokardialen Schädigung in ein Labor mit schneller Analysezeit versandt.

\section{Anamnese und körperliche Untersuchung}

Die Anamnese und körperliche Untersuchung wird zunächst auf die gravierenden Ursachen des Thoraxschmerzes fokussiert. Eine komplette differenzierte klinische Untersuchung kann erfolgen, wenn eine lebensbedrohliche Ursache des Thoraxschmerzes nach dieser symptomorientierten Erst-Untersuchung wenig wahrscheinlich ist. Im Hinblick auf die möglicherweise bestehende Indikation für eine Thrombolyse werden die Kontraindikationen zur Thrombolyse abgefragt.

\section{2-Kanal-EKG}

Das 12-Kanal-EKG erlaubt die sichere Diagnose eines ST-StreckenHebungsinfarktes (STEMI) und in Zusammenschau mit der Klinik die unmittelbare Therapieeinleitung. Auch bei neu aufgetretenem Linksschenkelblock und typischer Symptomatik sollte unmittelbar (Entscheidungszeit seit Aufnahme $<20$ Minuten!) die Revaskularisationstherapie (Lyse oder Kathetertherapie) eingeleitet werden.

Liegt weder eine signifikante ST-Streckenhebung $(\geq 0,1 \mathrm{mV}$ in den Extremitätenableitungen oder $\geq 0,2$ $\mathrm{mV}$ in zwei benachbarten Brustwandableitungen) noch ein neu aufgetretener Linksschenkelblock vor, so kann zunächst die Diagnose eines ST-Strecken-Hebungsinfarktes nicht gestellt werden und eine Lysetherapie ist dann nicht indiziert. Bei diesen Patienten müssen neben einer engmaschigen EKG-Kontrolle (kontinuierliche ST-Streckenanalyse oder in kurzen Abständen wiederholte 12-Kanal-EKGs) zur Sicherung der Infarktdiagnose die Laboruntersuchungen abgewartet werden.

\section{Laboruntersuchungen}

Die Resultate der bereits unmittelbar bei Klinikaufnahme durchgeführten Laboruntersuchungen sollten binnen maximal einer Stunde zur Verfügung stehen. Die für die Sicherung eines Myokardschadens notwendigen Analysen - vor allem kardiales Troponin - kann alternativ mit Hilfe eines Schnelltestes bereits auf der Notaufnahmestation erfolgen. Das Ergebnis der Laboruntersuchung muss in Relation zum Zeitpunkt des Schmerzbeginns und der stationären Aufnahme gesetzt werden, gegebenenfalls müssen engmaschige Kontrollen (mindestens einmal im Abstand von vier Stunden) erfolgen.

Nach der aktuellen Definition liegt bei einem Patienten mit dem klinischen Symptom der instabilen Angina pectoris, einem EKG ohne ST-Hebungen und dem Nachweis von ansteigenden und dann wieder abfallenden kardialen Markern ein Myokardinfarkt vor (Myokardinfarkt ohne ST-Hebung = NSTEMI). Der Nachweis eines NSTEMI muss zur Aufnahme auf die Überwachungsstation („Coronary Care“) oder Intensivstation und zur weiterführenden Diagnostik und Therapie führen.

\section{Echokardiographie}

Neben klinischen und anamnestischen Befunden, der EKG-Diagnostik und den oben genannten Laboranalysen hat im Rahmen der Akutdiagnostik von Patienten mit akutem Myokardinfarkt die Echokardiographie einen besonderen Stellenwert. Sie ermöglicht die sofortige bettseitige Diagnostik von Infarktfolgen im Sinne von regionalen Wandbewegungsstörungen oder mechanischen Infarktkomplikationen (z.B Papillarmuskelabriss, postinfarzieller Ventrikelseptumdefekt) und ist zur Abgrenzung von Differentialdiagnosen relevant. Es ist daher sinnvoll und empfehlenswert, die Echokardiographie in die Frühdiagnostik von Patienten mit dem Verdacht auf einen Myokardinfarkt einzubeziehen.

\section{- Therapie}

Die Therapie von Patienten mit einem Myokardinfarkt ist abhängig vom Schweregrad des akuten Koronarsyndroms und erfolgt in der Frühphase auf der Überwachungsoder Intensivstation.

\section{Basisbehandlung}

Die Behandlungsstrategien unterscheiden sich bei Patienten mit STEMI und NSTEMI. In beiden Fällen besteht die Basisbehandlung aus Analgesie, Sedierung, Thrombozytenfunktionshemmung, Antikoagulation, Sauerstoffgabe und Betablockertherapie.

Wichtigstes Therapieziel bei der Behandlung von Patienten mit akutem Myokardinfarkt im Sinne eines STEMI ist die Reperfusion des betroffenen Infarktareals entweder durch Lyse oder perkutane Katheterintervention $(\mathrm{PCI})$.

\section{Systemische Thrombolyse- therapie}

Die systemische Thrombolysetherapie ist als das am weitesten verbreitete Reperfusionsverfahren etabliert. Der klare Vorteil besteht in der ubiquitären Verfügbarkeit und einer recht hohen Erfolgsrate bei rechtzeitigem Einsatz. Bei geeigneter Befundkonstellation ist es möglich, bereits in der prähospitalen Phase des STEMI eine noch frühere und damit noch effizientere Revaskularisation zu erreichen. Das Konzept der prästationären Lysetherapie wird derzeit wieder intensiver überprüft und gehört heute in einigen Regionen zur täglichen Praxis der Infarktbehandlung. Die Lysetherapie kann ohne Nachteile für den Patienten mit der nachfolgenden invasiven Diagnostik und interventionellen Therapie kombiniert werden, sodass die frühe Thrombolyse kein konkurrierendes sondern ein komplementäres Reperfusionsverfahren zur Interventionsbehandlung darstellt.

Insbesondere in der sehr frühen Phase des Myokardinfarktes (binnen der ersten 2-3 Stunden) ist die Thrombolysetherapie der interventionellen Therapie gleichwertig, sodass hier bei fehlenden Kontraindikationen immer eine entsprechende pharmakologische Reperfusion versucht werden sollte.

\section{Perkutane kathetergestützte Behandlung}

Bedingt durch wesentliche Nachteile der systemischen Thrombolyse (viele Kontraindikationen, Blutungskomplikationen, relativ hohe Rever- 
schlussrate) wurde bereits früh eine perkutane kathetergestützte $\mathrm{Be}$ handlung (PCI) der verschlossenen infarktauslösenden Arterie versucht. Dieses Verfahren hat sich als hocheffektiv erwiesen, da nur sehr wenige Kontraindikationen gegen ein interventionelles Vorgehen bestehen und somit bis zu 90\% aller ins Krankenhaus aufgenommenen Patienten akut therapiert werden können. Bei etwa 90\% der behandelten Patienten kann durch die mechanische Infarktgefäßrevaskularisation der prognostisch bedeutsame ungehinderte Koronarfluss (TIMI 3) herbeigeführt werden. Heute erfolgt bei der Mehrzahl der Patienten in der Akutphase des Myokardinfarktes eine Stentimplantation kombiniert mit den modernen pharmakologischen Methoden der Thrombozytenfunktionshemmung.

\section{Vor- und Nachteile der interventionellen Infarkt- therapie}

Im Vergleich zur Thrombolysetherapie ist die Überlebenswahrscheinlichkeit nach PCI höher und die Häufigkeit von zerebralen Blutungskomplikationen, Reinfarkten sowie Postinfarktangina im weiteren Krankenhausverlauf reduziert. Außerdem ist bereits zum Zeitpunkt des Infarktes die Koronarmorphologie und Ventrikelfunktion des Patienten bekannt, sodass eine entsprechend begründete individuelle Differentialtherapie möglich ist.

Der entscheidende Nachteil der interventionellen Infarkttherapie ist der hohe Personal- und Materialaufwand, der nur in wenigen entsprechend ausgerüsteten Zentren angeboten werden kann. Heute haben in Deutschland etwa 600 Krankenhäuser eine kardiologische Fachabteilung, zirka 300 Kliniken sind mit einem Herzkatheterlabor ausgestattet. In etwa 70\% dieser Laboratorien werden Infarktgefäßinterventionen durchgeführt, allerdings nicht überall in 24-stündiger Dienstbereitschaft.

Bei Verfügbarkeit einer sofortigen Intervention in einem Krankenhaus mit Katheterlabor (d.h. in etwa 5-10\% aller deutschen Krankenhäuser) sollte schnellstmöglich (Reak- tionszeit < 60 Minuten!) die mechanische Infarktgefäßeröffnung erfolgen.

Wird ein Patient mit akutem Infarkt in ein Krankenhaus ohne Möglichkeit der mechanischen Intervention aufgenommen, so stellt sich die Frage, ob dieser Patient in ein interventionelles Zentrum zur Akutintervention verlegt werden soll. Aktuelle Empfehlungen raten zumindest bei Patienten mit Kontraindikationen gegen eine Thrombolyse und bei hämodynamisch instabilen Patienten zur sofortigen Verlegung und Intervention. Einige Untersuchungen sprechen sogar dafür, bei allen Patienten mit STEMI und einem Infarktalter von mehr als drei Stunden eine interventionelle Sofortbehandlung anzustreben. Vorraussetzung ist die Erreichbarkeit eines erfahrenen interventionellen Zentrums binnen 90 Minuten. Daher sollten regionale Versorgungsstrukturen geschaffen werden, in denen zwischen Krankenhäusern ohne Katheterlabor und professionellen interventionellen Zentren Kooperationen mit funktionierender Verlegungslogistik aufgebaut werden, um die flächendeckende optimale Versorgung von Patienten mit Myokardinfarkt zu ermöglichen.

\section{Funktionierende Verlegungs- logistik zwischen Kranken- häusern}

Bei Patienten mit NSTEMI ist eine Lysetherapie nicht indiziert, pharmakologisch wird bei fortbestehender Schmerzsymptomatik, fluktuierenden EKG-Veränderungen und hämodynamischer Instabilität zusätzlich zur oben genannten Basistherapie eine aggressive Thrombozytenfunktionshemmung durch Gabe von Glycoprotein IIb/IIIa-Hemmern empfohlen. Bei Patienten mit akutem Koronarsyndrom im Sinne der instabilen Angina pectoris oder eines NSTEMI wurde so in zahlreichen Studien eine Reduktion unerwünschter kardialer Ereignisse nachgewiesen.

Darüber hinaus konnte gezeigt werden, dass eine frühzeitige invasive Diagnostik und Therapie die Prognose von Patienten mit NSTEMI verbessert. Insbesondere in den ersten 24 Stunden nach Symptombeginn ist von einem erheblichen Therapieeffekt auszugehen, sodass die „First-Day-PCI“ heute bei diesen Patienten angestrebt werden sollte. Dies kann in einem funktionierenden regionalen Netzwerk flächendeckend angeboten werden.

\section{Summary}

of decisive importance for the acute management of myocardial infarction patients is the rapid and reliable diagnosis, correct stratification of risk and the fastest possible of appropriate treatment.

The basic therapy of myocardial infarction (NSTEMI and STEMI) comprises analgesia, sedation, platelet aggregation inhibitors, anticoagulation, administration of oxygen and beta blockers. In the case of STEMI, the primary objective is the revascularisation of the infracted vessels by thrombolysis, in particular in the first three post-infarction hours, where applicable in the prehospital setting, or catheterisation. In the case of STEMI, catheterisation is the most successful revascularisation method, but is of only limited availability. By establishing regional cooperation networks, interventional treatment of many patients with STEMI can be accomplished, so that the nationwide establishment of such care-providing structures between hospitals with no catheter laboratory and experienced interventional centres is both rational and necessary. Patients with NSTEMI also benefit from early (within 24 hours) invasive diagnostic work-up and interventional therapy, so that these patients too, should be managed within the framework of such a network.

Anschrift des Verfassers Prof. Dr. med. Matthias P. Heintzen Klinikum Braunschweig Medizinische Klinik II Klinik für Kardiologie, Pneumologie Angiologie Salzdahlumer Straße 90 38126 Braunschweig 